\title{
The $\mathbf{S}^{4} \mathrm{G}$ view of stellar mass, mid-IR dust, and evolved, intermediate-age stars in nearby galaxies
}

\author{
Sharon E. Meidt ${ }^{1}$ and $\mathbf{S}^{4} \mathbf{G}$ team \\ ${ }^{1}$ Max Planck Institute für Astronomie \\ Königstuhl 17, \\ DE-69117, Heidelberg, Germany \\ email: meidt@mpia.de
}

\begin{abstract}
With deep imaging at 3.6 and $4.5 \mu \mathrm{m}$ where the light in nearby galaxies is dominated by old stars, the Spitzer Survey of Stellar Structure in Nearby Galaxies $\left(S^{4} G\right)$ promises to be the ultimate inventory of stellar mass and structure in the local universe. We present results from a novel technique that makes it possible to fully exploit the information contained in these images, pertaining not only to the stellar light (and, ultimately, mass distribution), but also the nature and distribution of the mid-IR dust and the properties of evolved, intermediate age stars (e.g. in AGB-dominated star clusters). We apply Independent Component Analysis (ICA) to the 3.6 and $4.5 \mu \mathrm{m}$ bands to separate the light from the old stars from the secondary non-stellar (i.e. PAH and hot dust) sources of emission, which are identified via comparison to the nonstellar emission imaged at $8 \mu \mathrm{m}$. Then, within the context of age and mass estimation at high $z$, we extract optical-to-mid-IR SEDs for a sample of ICA-identified AGB-dominated clusters to constrain the typically uncertain fractional contribution of AGB light to the total stellar emission in (rest-frame) NIR bands.
\end{abstract}

Keywords. galaxies: structure, galaxies: stellar content, galaxies: star clusters, stars: AGB and post-AGB

\section{Introduction}

The Spitzer Survey of Stellar Structure in Galaxies ( ${ }^{4} \mathrm{G}$; roughly 2300 galaxies within $40 \mathrm{Mpc}$; Sheth et al. (2010)) provides an un-paralleled inventory of stellar mass and structure in nearby galaxies, with deep imaging at $3.6 \mu \mathrm{m}$ and $4.5 \mu \mathrm{m}$ (reaching $\mu_{3.6 \mu \mathrm{m}}(A B) \sim 27$ mag $\operatorname{arcsec}^{-2}$ ) where the light mainly traces the oldest stars. But alongside the $\mathrm{K}$ and $\mathrm{M}$ giants that dominate the flux in these two shortest IRAC wavebands, several other sources appear in star-forming galaxies: PAH (the $3.3 \mu \mathrm{m}$ emission feature in the $3.6 \mu \mathrm{m}$ band and the PAH continuum at $4.5 \mu \mathrm{m}$; Flagey et al. 2006), accessory non-stellar thermal continuum emission (i.e. from hot dust; Mentuch et al. 2009, 2010), and lower-M/L asymptotic giant branch (AGB) and red supergiant (RSG) stars can all make significant local contributions to the emission at these wavelengths.

To obtain a contamination-free view of the old stellar disk, we have developed a technique using ICA (FastICA; Hyvärinen (1999) and Hyvärinen \& Oja(2000)), which requires only the information from 3.6 and $4.5 \mu \mathrm{m}$ bands - the only data available for a large fraction of the sample - and accounts for the fact that dust and stellar SEDs have different shapes between these two wavelengths. Cleaned maps of the old stellar light exhibit [3.6]-[4.5] colors consistent with those of $\mathrm{K}$ and $\mathrm{M}$ giants (cf. Pahre et al. 2004; Hunter et al. 2006) and retain full 2D structural information, as demonstrated below for M100 (and see Figure 1). 

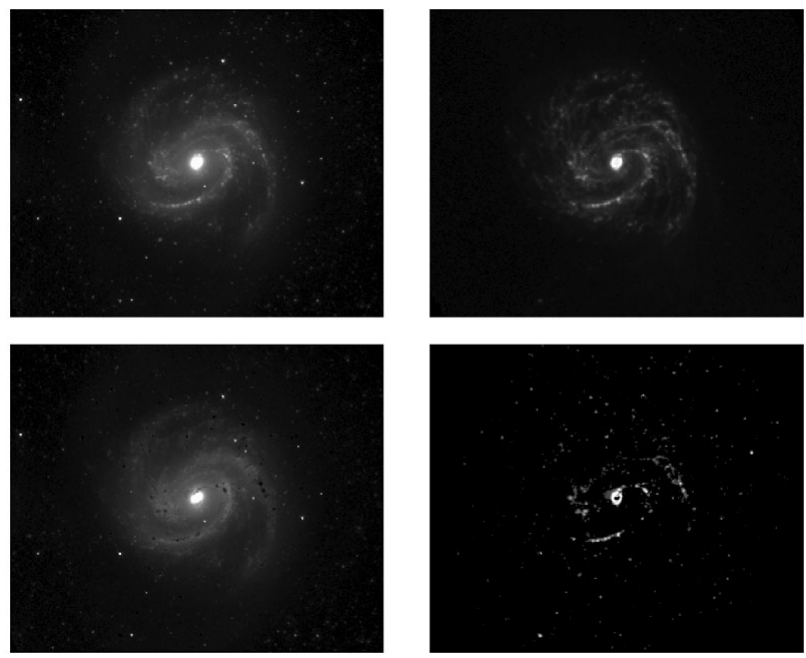

Figure 1. Clockwise from top left: $\mathrm{S}^{4} \mathrm{G}$ pipeline-processed IRAC $3.6 \mu \mathrm{m}$ image of M100 (Sheth et al. 2010); SINGS IRAC $8 \mu \mathrm{m}$ image (Kennicutt et al. 2003) shown with the stellar continuum subtracted; ICA s2 map of non-stellar (PAH and hot dust) contaminant emission at $3.6 \mu \mathrm{m}$; ICA s1 map of old stellar light at $3.6 \mu \mathrm{m}$ tracing the stellar mass. The map at the bottom left is a clean, smoothed version of the uncorrected image (top left), while the contaminant map (bottom right) contains most, if not all, of the bright and knotty features tracing star formation (cf. the PAH-dominated $8 \mu \mathrm{m}$ emission; top right). All images are shown on a square root intensity scale. Field Dimensions: 10.6 '×8.75'.

\section{ICA separation between old stars and dust}

The old stellar light Contaminant-free maps at $3.6 \mu \mathrm{m}$ compare well with other standard NIR tracers of the old stellar light, as shown in the top left panel of Figure 2. The H-[3.6] color profile for the cleaned map shows better agreement with the predictions from synthesized stellar SED fits to an old, dust-free elliptical for H-[3.6] 0.4. $\dagger$ The bluer colors reflect a localized reduction in the amount of light relative to the original maps occuring, i.e., in the spiral arms.

Sources of dust emission The quality in the map of the old stellar light stems from the ability of ICA to detect all sources of emission apart from the oldest stars: hot dust, $\mathrm{PAH}$ and bright but low $\mathrm{M} / \mathrm{L}$ intermediate-age stars. By comparing the ICA contaminant maps at $3.6 \mu \mathrm{m}$ to the non-stellar emission imaged at $8 \mu \mathrm{m}$ all three of these sources can be identified (see the top right panel of Figure 2). Non-stellar $3.6 \mu \mathrm{m}$ fluxes in M100 show a clear linear relation to $8 \mu \mathrm{m}$ fluxes over almost the full range in $F_{8, n s}$, with a slope that is consistent with the expected scaling $\alpha$ between the PAH emission at $3.3 \mu \mathrm{m}$ and at 8 $\mu m(0.03 \lesssim \alpha \lesssim 0.09$, as measured in the Milky Way; see Flagey et al. 2006 and references therein). Other galaxies exhibit a second, steeper relation $F_{3.6} / F_{8, n s} \sim 0.3$ characteristic of hot dust (Meidt et al. 2011a; less prominent in the particular case of M100).

A third source of emission can be identified as the thin, almost vertical concentration of points at the far left $\left(F_{8, n s} \lesssim 2 \mathrm{MJy} / \mathrm{sr}\right)$ in the top right plot in Figure 2. This emission arise from 'red knots' that are spatially distinct from the majority of the dust (see the bottom left panel of Figure 2) and populate a different region in the color-magnitude space at the bottom right in Figure 2: knots with $F_{s 2,3.6}>0.3 F_{8}$ extend to fainter magnitudes and have redder [3.6]-[4.5] colors (at a given magnitude) than dusty regions with $F_{s 2,3.6}<0.3 F_{8}$. The red colors of the former set are consistent AGB stars, in particular (the most luminous

\footnotetext{
$\dagger$ see http://web.ipac.caltech.edu/staff/jarrett/irac/calibration/galaxies.html
} 

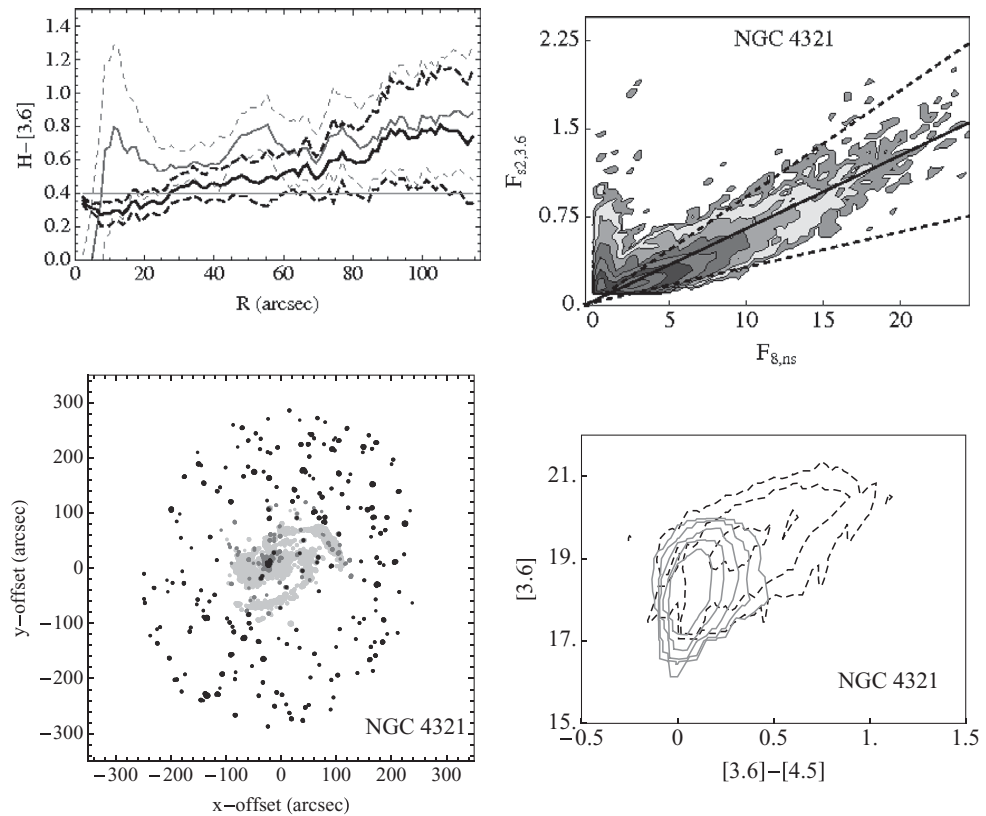

Figure 2. (Top left) H-[3.6] color profiles in M100 for the original and corrected maps shown in thin and thick black solid lines, respectively. Dashed errors show the range of values at each radius. The H-[3.6] color expected for an old, dust free population, $\mathrm{H}-[3.6]=0.4$ (see text), is shown as a gray horizontal line. (Top right) Contour plot of non-stellar $3.6 \mu \mathrm{m}$ fluxes $F_{s 2,3.6}$ vs. non-stellar $8 \mu \mathrm{m}$ fluxes $F_{8, n s}$. Overlaid dashed lines represent the expected range in $F_{s 2,3,6}=\alpha F_{8, n s}$ for PAH emission, with $\alpha=0.09$ or 0.03 (see text). A third solid line shows the best-fit linear relation with slope $\alpha=0.062$. (Bottom left) Map of pixels representative of the 'dust' and 'red knot' components in the ICA contaminant distribution. Emission from PAH (light gray), hot dust (dark gray) and 'red knots' (black) is selected from within the range $F_{s 2,3.6} / F_{8, n s}<0.09$, $0.1<F_{s 2,3.6} / F_{8, n s}<0.3$, and $F_{s 2,3.6} / F_{8, n s}>0.3$, respectively, in the plot at the top right. (Bottom right) CMD for pixels representative of the primary 'dust' $\left(F_{s 2,3.6}<0.3 F_{8, n s}\right.$; in gray solid line, including emission from both PAH and hot dust) and secondary 'red knot' $\left(F_{s 2,3.6}>0.3 F_{8, n s}\right.$; in black dashed line) components in the ICA contaminant distribution.

with $0.5<[3.6]-[4.5]<1$; Groenewegen (2006)), which dominate the light from all other stars at rest-frame wavelengths $\lambda \gtrsim 1 \mu \mathrm{m}$ between ages $0.2-1 \mathrm{Gyr}$.

\section{Circumstellar dust extinction of AGBs in M100}

The isolation of AGB-dominated clusters with ICA provides us with a novel opportunity to explore variation in the typically uncertain fractional contribution of AGB light to intermediate-age populations in (rest-frame) NIR bands. For a sample of 17 AGB-dominated clusters chosen from regions least susceptible to the effects of dust extinction/reddening, we extract optical-to-mid-IR SEDs using archival SINGS data and new HAWK-I JHK imaging (i.e. Grosbol \& Dottori 2011). We find that NIR brightness is coupled to the mid-IR dust emission such that a significant reduction of AGB light of up to $1 \mathrm{mag}$ in K-band follows from extinction by the dust shell formed during this stage (Meidt et al. 2011b). Our sample of clusters-each the analogue of a $\sim 1$ Gyr old post-starburst galaxy-has implications within the context of mass and age estimation via SED modelling at high $\mathrm{z}$ : we find that the average $\sim 0.5 \mathrm{mag}$ extinction estimated here may be sufficient to reduce the AGB contribution in (rest-frame) K-band from $\sim 70 \%$, as predicted in the latest generation of synthesis models, to $\sim 35 \%$ (see Figure 3 ). 

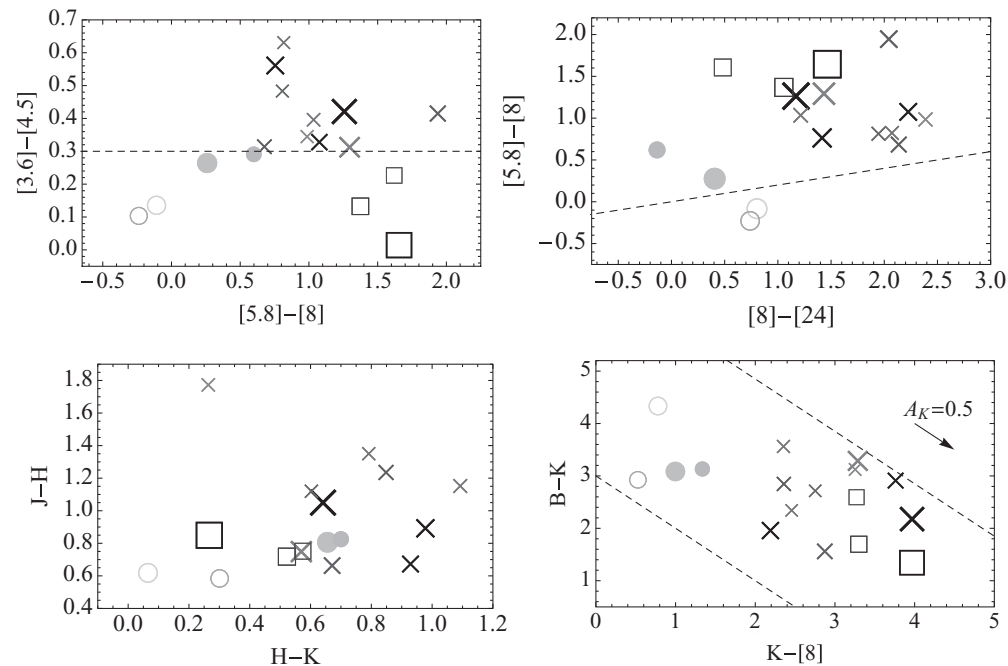

Figure 3. (Top left, right) Separation between clusters hosting C-rich (closed), O-rich (open) or extreme (crosses) AGB stars, as motivated by Boyer et al. 2011 (and see, e.g., Groenewegen 2006). Square symbols mark a fourth subset of 'mid-IR bright' objects that could potentially be dusty young clusters or HII regions (see Corbelli et al. 2011). The dashed black lines show our adopted criteria for distinguishing between types. The gray scaling shows variation in $\mathrm{B}-\mathrm{V}$ color, from $\mathrm{B}-\mathrm{V}=0.5$ (black) to $\mathrm{B}-\mathrm{V}=1.5$ (light gray), used here as a proxy for age, while the symbol size varies according to metallicity, from low (small) to high (large). (Bottom left) J-H vs. H-K for AGB-dominated clusters, with symbols and colors as above. (Bottom right) B-K vs. K-[8] colors showing the impact of the different AGB dust chemistries and mass loss rates. The B-K color traces the prominence of the AGB relative to the other (less evolved) cluster members that alone contribute in the optical. The $\mathrm{K}-[8]$ color serves as a measure of the amount of dust produced around each AGB. Clusters dominated by extreme AGBs preferentially lie at bluer B-K and redder K-8 colors than their O-rich and C-rich counterparts. The downward shift corresponds to on average 0.5-1.0 mag extinction in $\mathrm{K}$ at fixed V-band brightness.

\section{References}

Boyer, M., Srinivasan, S., van Loon, J. et al. 2011, AJ, 142, 103

Bruzual, G. \& Charlot, S. 2003, MNRAS, 344, 1000

Corbelli, E., Giovanardi, C., Palla, F., \& Verley, S. 2011, A\& $A, 528,116$

Flagey, N., Boulanger, F., Verstraete, L., et al. 2006, A\&A, 453, 969

Groenewegen, M. A. T. 2006, A\&\&A, 448, 181

Grosbol, P. \& Dottori, H. 2011, astro-ph/1109.4255

Hunter, D. A., Elmegreen, B. G., \& Martin, E. 2006, AJ, 132, 801

Hyvärinen A., 1999, IEEE Signal Processing Lett., 6, 145

Hyvärinen, A. \& Oja, E. 2000, Neural Networks, 13, 411

Kennicutt, Jr., R. C., et al. 2003, PASP, 115, 928

Meidt, S. et al. 2011a, ApJ in press

Meidt, S. et al. 2011b, in prep.

Mentuch, E., Abraham, R. G., Glazebrook, K., et al. 2009, ApJ, 706, 1020

Mentuch, E., Abraham R. G., Zibetti S. 2010, ApJ, 725, 1971

Pahre, M. A., Ashby, M. L. N., Fazio, G. G. \& Willner, S. P. 2004a, ApJS, 154, 229

Sheth, K, Regan, M., Hinz, J., et al. 2010, PASP, 122, 1397 